\title{
The 3-loop Beta Function of QCD
}

\author{
B. Allés ${ }^{\mathrm{a}}$, C. Christou ${ }^{\mathrm{b}}$, A. Feo ${ }^{\mathrm{a}, \mathrm{c}}$, H. Panagopoulos ${ }^{\mathrm{b} *}$ and E. Vicari ${ }^{\mathrm{a}}$

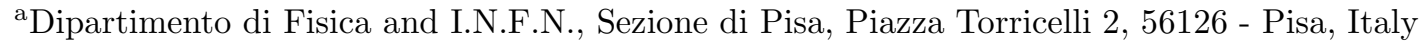 \\ ${ }^{\mathrm{b}}$ Department of Natural Sciences, University of Cyprus, P.O. Box 537, Nicosia CY-1678, Cyprus \\ ${ }^{\mathrm{c} S c u o l a ~ N o r m a l e ~ S u p e r i o r e, ~ P i s a, ~ I t a l y ~}$
}

Using the background field technique, we calculate the 3-loop beta function of lattice $S U(N)$ gauge theories. In the pure gluonic case, we present our results, comparing to those recently obtained by Luescher and Weisz. We also provide a progress report in the case of QCD with Wilson fermions.

\section{INTRODUCTION}

The relationship between the bare coupling and the cutoff is an essential ingredient in lattice calculations. Usual Monte Carlo simulations require the knowledge of this relationship far from the critical point, where corrections to asymptotic scaling could become relevant.

In order to check the relevance of these scaling corrections, we present here a calculation of the first non-universal coefficient of the lattice $\beta$ function in pure Yang-Mills theory with Wilson action [1, as well as in the presence of Wilson fermions 2. This renormalization group function can be written as

$\beta^{L}\left(g_{0}\right) \equiv-\left.a \frac{\mathrm{d} g_{0}}{\mathrm{~d} a}\right|_{g_{r}, \mu}=-b_{0} g_{0}^{3}-b_{1} g_{0}^{5}-b_{2}^{L} g_{0}^{7}-\cdots$

where $a$ is the lattice spacing, $g_{0}$ the lattice bare coupling, $g_{r}$ is the renormalized coupling constant and $\mu$ the subtraction point. It establishes how the bare coupling and the cutoff $a$ must simultaneously vary to keep renormalized quantities fixed. The first two coefficients in eq. (1) are known as they are scheme-independent. Our purpose is to compute the third coefficient, $b_{2}^{L}$.

In a recent paper [3] the authors calculated a quantity related to this coefficient (in the pure gluon case), using a coordinate space method [4] for evaluating lattice integrals. In our calculation we have used a different technique where superficially divergent integrands are Taylor expanded

\footnotetext{
*Presented the talk.
}

in $D$ dimensions. The idea is a generalization to two loops of the procedure introduced in [5]. Thus, our results for the pure gluon case provide an independent check of the calculation in reference [3].

In what follows we will first define the quantities we set out to compute, and give a brief description of our calculational techniques. We then present our results. We discuss the effect of $b_{2}^{L}$ on the scaling behaviour of dimensionful quantities. We close with some concluding remarks.

The involved algebra of lattice perturbation theory was carried out by means of a computer code. The main features of this code were outlined in [6] and used to compute the three loop perturbative background of the topological susceptibility [6], and the three loop lattice free energy [7], in pure Yang-Mills on the lattice. For the purpose of the present work, this code was extended to include form factors, and to handle fermionic vertices.

Aside from the intrinsic interest of the result, this calculation serves also as a prototype for numerous 2-loop calculations involving fermions and external momenta, e.g. multiplicative renormalization of fermionic currents. We expect to return to this issue in a future report.

\section{GENERAL CONSIDERATIONS}

We performed our calculation using the background field method. To facilitate comparison, we have adopted the notation of Ref.[3]. 
We want to calculate $\nu^{(2)}(p)$, defined through the lattice background field two point function via:

$$
\sum_{\mu} \Gamma^{(2,0,0)}(p,-p)_{\mu \mu}^{a b}=-\delta^{a b} 3 \hat{p}^{2}[1-\nu(p)] / g_{0}^{2}
$$

with $\nu(p)=\sum g_{0}^{2 l} \nu^{(l)}(p)$. Knowledge of $\nu^{(2)}(p)$, together with its $\overline{M S}$ counterpart $\nu_{R}^{(2)}(p)$ and the one loop gluon self energy leads us, through use of standard formulae, to $Z\left(g_{0}, \mu a\right) \quad\left(g_{0}=\right.$ $\left.Z\left(g_{0}, \mu a\right) g(\mu)\right)$. Knowledge of the three loop coefficient of the renormalized $\beta$-function, $b_{2}^{\overline{M S}}$, along with the two loop coefficient $Z_{20}$ in the expansion:

$Z\left(g_{0}, \mu a\right)=1+\sum_{i=1} \sum_{j=1}^{i} g_{0}^{2 i} Z_{i j} \ln ^{j} \mu a$

gives us the three loop lattice $\beta$ function, through the relation:

$b_{2}^{L}=b_{2}^{\overline{M S}}-2 b_{1} Z_{10}+b_{0} Z_{10}^{2}+2 b_{0} Z_{20}$

All one loop quantities appearing above are well known. For the continuum function $\nu_{R}^{(2)}(p)$, in the presence of $N_{f}$ fermionic species, we find (in the Feynman gauge):

$$
\begin{aligned}
& \nu_{R}^{(2)}(p)\left(16 \pi^{2}\right)^{2}=N^{2}[8 \rho+577 / 18-6 \zeta(3)] \\
& +N_{f}[(-3 \rho-401 / 36) N+(\rho+55 / 12-4 \zeta(3)) / N] \\
& \left(\rho=\ln \left(\mu^{2} / p^{2}\right)\right) .
\end{aligned}
$$

Contributions to $\nu^{(2)}(p)$ come from 35 diagrams in the pure gluonic case (shown in Ref.[3]). Fermions bring in 18 more two-loop diagrams (the ghost diagrams of [3], with fermions replacing ghosts) plus 2 one-loop diagrams and 2 diagrams with fermion mass counterterms (we work with zero renormalized fermion mass). We manipulate these diagrams using a computer code $[6]$ which has now been extended to include fermionic vertices and external momenta. The code starts with a series of operations which can be performed on diagrams with arbitrary number/type of vertices/loops/external legs: Generation of vertices, contraction with appropriate reassignment of indices, symmetrization, reduction of Dirac and color indices, simplification of Lorentz structures. It is essential that the corresponding algorithms be (quasi-)polynomial: The contrary results in astronomical requirements of $\mathrm{CPU}$ and RAM, even in the simplest cases. This last constraint turns out to be the most exacting one in the construction of our algorithms.

At this point, we apply trigonometry judiciously to bring our expressions into a "canonical" form, separating them also into (a few) superficially divergent terms plus the remainder. This remainder can be quite sizeable, especially in the fermionic case (often thousands of terms) but it can be handled with a straightforward Taylor expansion in external momenta or, when subdivergences are present, with appropriate subtractions; e.g., in

$(2 \pi)^{-8} \int d^{4} q d^{4} k \stackrel{\circ}{q} \cdot \stackrel{\circ}{k} \stackrel{\circ}{q} \cdot \stackrel{\circ}{p} /\left(\hat{q}^{2} \hat{k}^{2} \widehat{q+k}^{2} \widehat{k+p}^{2}\right)$

the subtraction: $1 / \widehat{q+k}^{2}=1 / \hat{q}^{2}+\left(1 / \widehat{q+k}^{2}-1 / \hat{q}^{2}\right)$ leads to a product of one loop integrals with a single $\ln p$, plus a Taylor expandable part. Different types of subtractions may be necessary for the full evaluation of a diagram; suffice it to say that the most cumbersome diagram (a "diamond" with a triangle of fermions) splits into 100 types of expressions.

For the superficially divergent diagrams, we have extended beyond one loop the proceduce of Ref. [5] (analytic continuation to $4-2 \epsilon$ dimensions, followed by Taylor expansion). This entails a lengthy series of additions/subtractions to parts of the integrand and to the integration domain (so that, e.g., $1 / \widehat{k+q}^{2}=1 / \hat{q}^{2}+\ldots, 1 / \hat{q}^{2}=1 / q^{2}+\ldots$, $[-\pi / a, \pi / a]^{4}=R^{4}-\ldots$, etc.). Interchanging the $\epsilon \rightarrow 0$ and $a \rightarrow 0$ limits is more complicated in this case. Also, certain intermediate expressions must be calculated to $\mathcal{O}(\epsilon)$. Proceeding in this way we have produced [1] a table of values for all such two-loop diagrams, complementing those given in [3].

Our numerical integrations were done on finite lattices, with subsequent extrapolation to infinity, using the form $\sum_{m, n} a_{m n}\left(\ln ^{m} L\right) / L^{n}$ ( $L$ : lattice size). This leads typically to four significant digits. The coordinate space method of Ref. a much better precision, but cannot be applied as is to fermionic diagrams. 


\section{RESULTS}

The contribution $\nu_{i}(p)$ of the $i$ th diagram to $\nu^{(2)}(p)$ is:

$$
\begin{gathered}
\hat{p}^{2} \nu_{i}(p)=c_{0, i}+c_{1, i} \sum_{\mu=0}^{3} \frac{p_{\mu}^{4}}{p^{2}}+p^{2}\left\{c_{2, i}\left[\ln p^{2} /(4 \pi)^{2}\right]^{2}\right. \\
\left.+c_{3, i} \ln p^{2} /(4 \pi)^{2}+c_{4, i}\right\}+\mathcal{O}\left(p^{4}\right)
\end{gathered}
$$

where: $c_{n, i}=c_{n, i}^{(0)} / N^{2}+c_{n, i}^{(1)}+c_{n, i}^{(2)} N^{2}$ for diagrams without fermions and $c_{n, i}=c_{n, i}^{(0)} / N+c_{n, i}^{(1)} N$ for diagrams with fermions.

Gauge invariance requires that $\sum_{i} c_{0, i}=0$; this is indeed so, separately for diagrams with and without fermions, as we have checked both by numerical evaluation and algebraically (expressing all vertices involved in terms of derivatives of the gluon and fermion propagators, and performing integration by parts where necessary). Lorentz invariance requires: $\sum_{i} c_{1, i}=0$. Such contributions appear in non-tadpole diagrams containing one-loop renormalized gluon propagators; again, we verified that their sum vanishes, both numerically and algebraically. The coefficients $c_{2, i}$ of the logarithms squared must coincide with those of the corresponding continuum diagrams and indeed they do.

In the pure gluonic case, we compared our results to those of Lüscher and Weisz. All coefficients $c_{0, i}, c_{1, i}, c_{2, i}, c_{3, i}$ coincide. As for $c_{4, i}$, the parts $c_{4, i}^{(0)}$ and $c_{4, i}^{(1)}$ agree for each diagram. The coefficients $c_{4, i}^{(2)}$ agree for all but 1 diagram (\# 5 of [3]), leading to a slightly smaller $(<3 \%)$ total for $c_{4}^{(2)}$. To this writing, we have not been able to find the cause of this discrepancy.

Thus, comparing with the expression of [3] for $\nu^{(2)}(p)$ without fermions:

$$
\begin{aligned}
\nu^{(2)}(p)= & -N^{2} /\left(32 \pi^{4}\right) \ln p^{2}+1 / N^{2}(3 / 128) \\
& -0.01654462+N^{2} 0.0074438722(8)
\end{aligned}
$$

we find for the last number in the above: 0.007230 .

Our results for $\nu^{(2)}(p)$ with Wilson fermions are in the final stage of extrapolation/precision checks [2].

\section{CONCLUSIONS}

Eq. (8) for $\nu^{(2)}(p)$ can be used directly to evaluate $b_{2}^{D}$, as explained. In the absence of fermions, we find:

$\left(16 \pi^{2}\right)^{3} b_{2}^{L}=-2143 / N+1433.8 N-366.2 N^{3}$

Substituting now in the asymptotic scaling formula:

$$
\begin{aligned}
a \Lambda_{L}= & \exp \left(-1 / 2 b_{0} g_{0}^{2}\right)\left(b_{0} g_{0}^{2}\right)^{-b_{1} / 2 b_{0}^{2}} . \\
& \left(1+g_{0}^{2}\left(b_{1}^{2}-b_{2}^{L} b_{0}\right) / 2 b_{0}^{3}+\ldots\right)
\end{aligned}
$$

the last factor becomes, for $S U(3)$ :

$\left(1+0.1896 g_{0}^{2}\right)$

Thus, in the region $g_{0} \sim 1$ this factor brings about a rather sizeable correction to asymptotic scaling.

Some further computations which can be carried out without changes in our computer code are two loop renormalization of various composite operators (fermionic currents, etc.), use of improved actions and (with some modifications) use of staggered fermions.

\section{REFERENCES}

1. B. Allés, A. Feo and H. Panagopoulos, The Three-Loop $\beta$ Function in $S U(N)$ Lattice Gauge Theories, Pisa-Cyprus preprint UCYPHY 96/9.

2. C. Christou, A. Feo, H. Panagopoulos and E. Vicari, The Three-Loop $\beta$ Function in $Q C D$ with Wilson Fermions, Pisa-Cyprus preprint IFUP-TH 50/96, UCY-PHY 96/11.

3. M. Lüscher and P. Weisz, Nucl. Phys. B452 (1995) 234.

4. M. Lüscher and P. Weisz, Nucl. Phys. B445 (1995) 429.

5. H. Kawai, R. Nakayama and K. Seo, Nucl. Phys. B189 (1981) 40.

6. B. Allés, M. Campostrini, A. Feo and H. Panagopoulos, Nucl. Phys. B413 (1994) 553.

7. B. Allés, M. Campostrini, A. Feo and H. Panagopoulos, Phys. Lett. B324 (1994) 433. 